\title{
Characterizing the magnetic field and spectral variability of the rigidly rotating magnetosphere star HD 345439
}

\author{
S. Hubrig ${ }^{1 \star}$, A. F. Kholtygin ${ }^{2}$, M. Schöller ${ }^{3}$, I. Ilyin ${ }^{1}$ \\ ${ }^{1}$ Leibniz-Institut für Astrophysik Potsdam (AIP), An der Sternwarte 16, 14482 Potsdam, Germany \\ ${ }^{2}$ Saint-Petersburg State University, Universitetskij pr. 28, Saint-Petersburg 198504, Russia \\ ${ }^{3}$ European Southern Observatory, Karl-Schwarzschild-Str. 2, 85748 Garching, Germany
}

Accepted Received; in original form

\begin{abstract}
A team involved in APOGEE, one of the Sloan Digital Sky Survey III programs, recently announced the discovery of two rare rigidly rotating magnetosphere stars, HD 345439 and HD 23478. Near-infrared spectra of these objects revealed emission-line behavior identical to that previously discovered in the helium-strong star $\sigma$ Ori $\mathrm{E}$, which has a strong magnetic field and rotates fast. A single spectropolarimetric observation of HD 345439 with FORS 2 at the VLT in 2014 over 88 min indicated that HD 345439 may host a strong, rapidly varying magnetic field. In this work, we present the results of our spectropolarimetric monitoring of this star with FORS 2, which revealed the presence of a strong longitudinal magnetic field dominated by a dipolar component. The analysis of spectral variability indicates an opposite behaviour of $\mathrm{He}$ and $\mathrm{Si}$ lines, which is usually attributed to differences in the distribution of surface $\mathrm{He}$ and $\mathrm{Si}$ abundance spots.
\end{abstract}

Key words: stars: early type — stars: individual: HD 345439 — stars: magnetic field stars: chemically peculiar — stars: abundances — techniques: polarimetric

\section{INTRODUCTION}

The presence of a rigidly rotating magnetosphere in the early B-type stars HD 23478 and HD 345439 was recently discovered in the Apache Point Observatory Galactic Evolution Experiment (APOGEE; Eikenberry et al. 2014) using high-resolution $(R \sim$ 22 500) near-infrared H-band spectra. The authors detected in the APOGEE bandpass prominent Brackett series emission lines with a characteristic double-horned profile. The type of profile and peak separation is typical for the rigidly rotating magnetosphere (RRM, Townsend \& Owocki 2005) feature previously discovered in the fast rotating helium-strong star $\sigma$ Ori E, which possesses an extremely large magnetic field (e.g., Landstreet \& Borra 1978; Oksala et al. 2015). Such stars are extremely rare: the discovery of HD 23478 and HD 345439 has enhanced the number of "extreme" rotators by $50 \%$ (Eikenberry et al. 2014). The authors reported that the optical spectra of HD 345439 reveal strong $\mathrm{He}_{\mathrm{I}}$ lines and very fast rotation of $\sim 270 \pm 20 \mathrm{~km} \mathrm{~s}^{-1}$ Subsequently, Wisniewski et al. (2015) analysed multi-epoch photometric observations of this star from the Kilodegree Extremely Little Telescope, Wide Angle Search for Planets, and ASAS surveys revealing the presence of a $\sim 0.7701$ day rotation period in each data set. The authors suggest that the He-strong star HD 345439 of spectral type $\mathrm{B} 2 \mathrm{~V}$ is among the faster known He-strong $\sigma$ Ori $\mathrm{E}$ analogs,

\footnotetext{
^ E-mail: shubrig@aip.de
}

$\operatorname{HR} 7355\left(P_{\text {rot }}=0.52 d-\right.$ Rivinius et al. 2013 $)$ and HR $5907\left(P_{\text {rot }}=\right.$ $0.51 d-$ Grunhut et al. 2012).

Hubrig et al. (2015a) carried out a spectropolarimetric followup of HD 345439 on one occasion, obtaining eight subexposures over 88 minutes in 2014 June with the FOcal Reducer low dispersion Spectrograph (FORS 2; Appenzeller et al. 1998) mounted on the $8 \mathrm{~m}$ Antu telescope of the VLT. The authors reported that the mean longitudinal magnetic field was changing from about $+500 \mathrm{G}$ measured in the first pair of subexposures to about $-1200 \mathrm{G}$ measured in the last pair of subexposures. Multi-epoch FORS 2 spectropolarimetric observations distributed over about two months were recently obtained in service mode in the framework of our programme 097.D-0428. In the following sections, we present the results of our magnetic fields measurements, the search for a magnetic field periodicity, and discuss the detected spectral variability with respect to the magnetic field geometry.

\section{OBSERVATIONS AND MAGNETIC FIELD MEASUREMENTS}

Fourteen FORS 2 spectropolarimetric observations of HD 345439 were obtained from 2016 May 17 to 2016 July 22. The FORS 2 multi-mode instrument is equipped with polarisation analysing optics comprising super-achromatic half-wave and quarter-wave phase retarder plates, and a Wollaston prism with a beam divergence of $22^{\prime \prime}$ in standard resolution mode. We used the GRISM 
Table 1. Logbook of the FORS 2 polarimetric observations of HD 345439, including the modified Julian date of mid-exposure followed by the achieved signalto-noise ratio in the Stokes $I$ spectra around $5200 \AA$, and the measurements of the mean longitudinal magnetic field using the Monte Carlo bootstrapping test, for the hydrogen lines and all lines. In the last columns, we present the results of our measurements using the null spectra for the set of all lines and the phases calculated relative to the zero phase corresponding to a positive field extremum at MJD56925.5425. All quoted errors are $1 \sigma$ uncertainties.

\begin{tabular}{crrrrr}
\hline \hline MJD & $\begin{array}{r}\text { SNR }_{5200} \\
\end{array}$ & \multicolumn{1}{c}{\begin{tabular}{c}
\multicolumn{1}{c}{$\left[B_{\mathrm{z}}\right\rangle_{\text {hydr }}$} \\
{$[\mathrm{G}]$}
\end{tabular}} & \multicolumn{1}{c}{\begin{tabular}{c}
\multicolumn{1}{c}{$\left[B_{\mathrm{Z}}\right\rangle_{\text {all }}$} \\
[G
\end{tabular}} & \multicolumn{1}{c}{$\begin{array}{c}\left\langle B_{\mathrm{Z}}\right\rangle_{\mathrm{N}} \\
{[\mathrm{G}]}\end{array}$} & Phase \\
\hline 56810.2547 & 413 & $414 \pm 282$ & $436 \pm 212$ & & 0.311 \\
56810.2745 & 455 & $789 \pm 246$ & $565 \pm 188$ & & 0.336 \\
56810.2860 & 392 & $-303 \pm 282$ & $-298 \pm 212$ & & 0.352 \\
56810.3018 & 420 & $-840 \pm 262$ & $-689 \pm 198$ & & 0.372 \\
57525.2797 & 521 & $1202 \pm 552$ & $1310 \pm 374$ & $-275 \pm 223$ & 0.697 \\
57527.2841 & 729 & $287 \pm 302$ & $416 \pm 206$ & $-29 \pm 166$ & 0.300 \\
57530.3230 & 960 & $1714 \pm 245$ & $1237 \pm 186$ & $-181 \pm 122$ & 0.246 \\
57530.3753 & 1086 & $514 \pm 185$ & $518 \pm 141$ & $-5 \pm 104$ & 0.314 \\
57531.2763 & 756 & $-829 \pm 408$ & $-475 \pm 222$ & $-274 \pm 274$ & 0.483 \\
57531.3233 & 811 & $-103 \pm 371$ & $-576 \pm 203$ & $76 \pm 205$ & 0.544 \\
57534.3069 & 786 & $-853 \pm 280$ & $-926 \pm 181$ & $123 \pm 194$ & 0.418 \\
57560.1708 & 881 & $3415 \pm 344$ & $3044 \pm 235$ & $113 \pm 178$ & 0.000 \\
57590.1750 & 1127 & $2546 \pm 184$ & $2551 \pm 121$ & $130 \pm 97$ & 0.957 \\
57590.2287 & 1174 & $1905 \pm 200$ & $2176 \pm 129$ & $-98 \pm 95$ & 0.027 \\
57590.2812 & 1056 & $2084 \pm 292$ & $2156 \pm 169$ & $-45 \pm 113$ & 0.095 \\
57591.1437 & 1053 & $1344 \pm 265$ & $1280 \pm 173$ & $-6 \pm 145$ & 0.215 \\
57591.1997 & 1178 & $826 \pm 199$ & $583 \pm 137$ & $-21 \pm 91$ & 0.288 \\
57591.2521 & 1133 & $-372 \pm 229$ & $-314 \pm 149$ & $-51 \pm 115$ & 0.356 \\
\hline
\end{tabular}

600B and the narrowest available slit width of $0 . ' 4$ to obtain a spectral resolving power of $R \sim 2000$. The observed spectral range from 3250 to $6215 \AA$ includes all Balmer lines, apart from $\mathrm{H} \alpha$, and numerous helium lines. For the observations, we used a non-standard readout mode with low gain $(200 \mathrm{kHz}, 1 \times 1$, low $)$, which provides a broader dynamic range, hence allowed us to reach a higher signalto-noise ratio (SNR) in the individual spectra. The exposure time for each subexposure accounted for $7.8 \mathrm{~min}$.

A first description of the assessment of longitudinal magnetic field measurements using FORS $1 / 2$ spectropolarimetric observations was presented in our previous work (e.g. Hubrig et al. $2004 \mathrm{a}$ b, and references therein). To minimize the cross-talk effect, and to cancel errors from different transmission properties of the two polarised beams, a sequence of subexposures at the retarder position angles $-45^{\circ}+45^{\circ},+45^{\circ}-45^{\circ},-45^{\circ}+45^{\circ}$, etc. is usually executed during the observations. Moreover, the reversal of the quarter wave plate compensates for fixed errors in the relative wavelength calibrations of the two polarised spectra. According to the FORS User Manual, the $V / I$ spectrum is calculated using:

$\frac{V}{I}=\frac{1}{2}\left\{\left(\frac{f^{\mathrm{o}}-f^{\mathrm{e}}}{f^{\mathrm{o}}+f^{\mathrm{e}}}\right)_{-45^{\circ}}-\left(\frac{f^{\mathrm{o}}-f^{\mathrm{e}}}{f^{\mathrm{o}}+f^{\mathrm{e}}}\right)_{+45^{\circ}}\right\}$

where $+45^{\circ}$ and $-45^{\circ}$ indicate the position angle of the retarder waveplate and $f^{\circ}$ and $f^{\mathrm{e}}$ are the ordinary and extraordinary beams, respectively. Rectification of the $V / I$ spectra was performed in the way described by Hubrig, Schöller, \& Kholtygin (2014). Null profiles, $N$, are calculated as pairwise differences from all available $V$ profiles. From these, $3 \sigma$-outliers are identified and used to clip the $V$ profiles. This removes spurious signals, which mostly come from cosmic rays, and also reduces the noise. A full description of the updated data reduction and analysis will be presented in a separate paper (Schöller et al., in preparation, see also Hubrig, Schöller, \& Kholtygin 2014). The mean longitudinal magnetic field, $\left\langle B_{\mathrm{z}}\right\rangle$, is measured on the rectified and clipped spectra based on the relation following the method suggested by

\section{Angel \& Landstreet (1970)}

$\frac{V}{I}=-\frac{g_{\text {eff }} e \lambda^{2}}{4 \pi m_{\mathrm{e}} c^{2}} \frac{1}{I} \frac{\mathrm{d} I}{\mathrm{~d} \lambda}\left\langle B_{\mathrm{z}}\right\rangle$,

where $V$ is the Stokes parameter that measures the circular polarization, $I$ is the intensity in the unpolarized spectrum, $g_{\text {eff }}$ is the effective Landé factor, $e$ is the electron charge, $\lambda$ is the wavelength, $m_{\mathrm{e}}$ is the electron mass, $c$ is the speed of light, $\mathrm{d} I / \mathrm{d} \lambda$ is the wavelength derivative of Stokes $I$, and $\left\langle B_{z}\right\rangle$ is the mean longitudinal (line-ofsight) magnetic field.

The longitudinal magnetic field was measured in two ways: using the entire spectrum including all available lines, excluding lines in emission, or using exclusively hydrogen lines. Furthermore, we have carried out Monte Carlo bootstrapping tests. These are most often applied with the purpose of deriving robust estimates of standard errors. The measurement uncertainties obtained before and after the Monte Carlo bootstrapping tests were found to be in close agreement, indicating the absence of reduction flaws.

Since the presence of $\beta$ Cep-like pulsations is frequently found in early B-type stars, we also checked the stability of the spectral lines along full sequences of sub-exposures. We have compared the profiles of several spectral lines recorded in the parallel beam with the retarder waveplate at $+45^{\circ}$. The same was done for spectral lines recorded in the perpendicular beam. The line profiles looked identical within the noise.

The results of our magnetic field measurements, those for the entire spectrum or only the hydrogen lines are presented in Table 1 where we also include in the first four rows the information about the previous magnetic field measurements presented by Hubrig et al. (2015a). A non-detection was obtained by the authors, if all four consecutive observations recorded as pairs of position angles separated by $90^{\circ}$ were combined. On the other hand, after splitting the observations into two data sets, i.e. using the first two pairs and the second two pairs consisting of observations at the retarder waveplate positions $\left(-45^{\circ},+45^{\circ},+45^{\circ},-45^{\circ}\right)$, they obtained 3.0 to $3.8 \sigma$ detections, but with $\left\langle B_{z}\right\rangle$ values with opposite sign, in- 


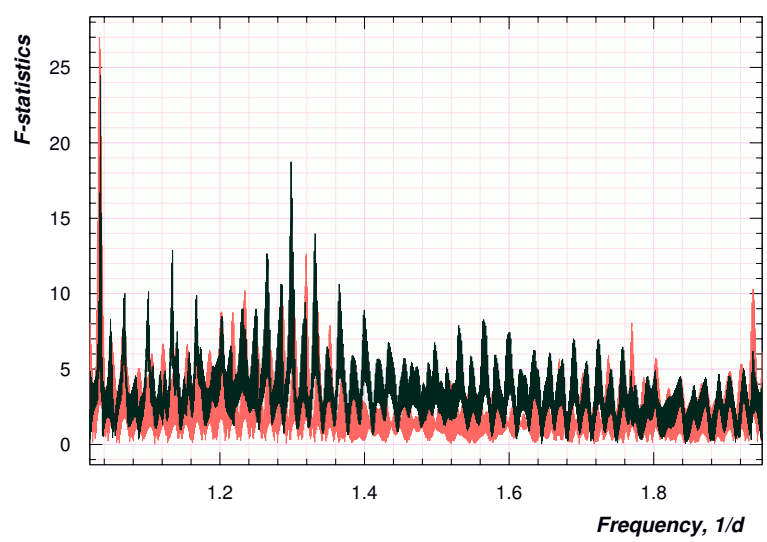

Figure 1. Frequency periodogram (in $\mathrm{d}^{-1}$ ) for the longitudinal magnetic field measurements of HD 345439 using both the entire spectrum and only the hydrogen lines. The window function is indicated by the red color.

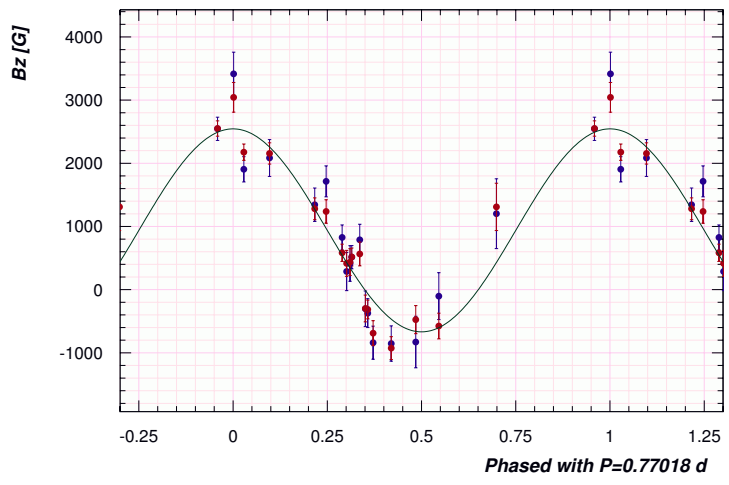

Figure 2. Longitudinal magnetic field variation of HD 345439 phased with the $0.77018 \mathrm{~d}$ period. Measurements of the magnetic field using the entire spectrum are presented by red circles, and those using hydrogen lines by blue circles. The solid line represents a fit to the data with a mean value for the magnetic field of $\overline{\left\langle B_{\mathrm{z}}\right\rangle}=939 \pm 96 \mathrm{G}$ and an amplitude of $A_{\left\langle B_{\mathrm{Z}}\right\rangle}=$ $1607 \pm 99 \mathrm{G}$. For the presented fit, we assume a zero phase corresponding to a positive field extremum at MJD56925.5425.

dicating a very fast rotation of HD 345439. The measurements in Table 1 refer to observations at just two position angles with a time lap of $22 \mathrm{~min}$. In this case, the null profile cannot be extracted. The rotation phase presented in the last column of Table 1 was calculated assuming a period of $0.77018 \mathrm{~d}$, which was determined from our period search described in Sect. 3 .

\section{PERIOD DETERMINATION FROM THE MAGNETIC DATA}

Magnetic early B-type stars usually exhibit photometric, spectral, and magnetic variability with the rotation period (e.g. Landstreet \& Borra 1978). Using Kilodegree Extremely Little Telescope (KELT) photometry, Wisniewski et al. (2015) detected a rotation period of $0.7701 \pm 0.0003 \mathrm{~d}$ with the zero point of the ephemeris $J D=2454252.3432$ corresponding to the first minimum in the light curve (see their Fig. 1.). From archival photometric observations in the Wide Angle Search for Planets (SuperWASP) survey and the All Sky Automated Survey (ASAS), the authors derived $P_{\text {rot }}=0.7695 \pm 0.0078 \mathrm{~d}$ and $P_{\text {rot }}=0.7702 \pm 0.0001 \mathrm{~d}$, respectively, with the phase-folded light curves exhibiting similar complex morphology.

The result of our frequency analysis based on the longitudinal magnetic field measurements presented in Table 1 and performed using a non-linear least squares fit to the multiple harmonics utilizing the Levenberg-Marquardt method (Press et al. 1992) with an optional possibility of pre-whitening the trial harmonics is presented in Fig. 1. Since the results of the measurements using the whole spectrum or exclusively the hydrogen lines are rather similar, the frequency analysis was performed using both, the measurements on the entire spectrum and on the hydrogen lines. To detect the most probable period, we calculated the frequency spectrum and for each trial frequency we performed a statistical F-test of the null hypothesis for the absence of periodicity (Seber 1977). The resulting F-statistics can be thought of as the total sum including covariances of the ratio of harmonic amplitudes to their standard deviations, i.e. a signal-to-noise ratio. The highest peak in the periodogram not coinciding with the window function is detected at a frequency of $1.298 \mathrm{~d}^{-1}$. Using this value as an initial guess for a least-squares fit of the period, we obtain a value of $0.77018 \pm 0.00002 \mathrm{~d}$. This period is in good agreement with the results of the period search by Wisniewski et al. (2015) using photometric data.

In Fig. 2 we present all measurements, those using the entire spectrum and those using only the hydrogen lines, phased with the rotation period and the best sinusoidal fit calculated for these measurements. The largest gap in the phase coverage occurs in the phase range between 0.70 and 0.95 . From the sinusoidal fit to our data, we obtain a mean value for the variable longitudinal magnetic field $\overline{\left\langle B_{\mathrm{z}}\right\rangle}=939 \pm 96 \mathrm{G}$, an amplitude of the field variation $A_{\left\langle B_{z}\right\rangle}=1607 \pm 99 \mathrm{G}$, and a reduced $\chi^{2}$ value of 3.1. For the presented fit, we assume a zero phase corresponding to a positive field extremum at MJD56925.5425 \pm 0.0015 . The observed sinusoidal modulation indicates that the magnetic field structure exhibits two poles and a symmetry axis, tilted with respect to the rotation axis. The simplest model for this magnetic field geometry is based on the assumption that the studied stars are oblique dipole rotators, i.e., their magnetic field can be approximated by a dipole with its magnetic axis inclined to the rotation axis. In Fig. 2] we observe around the rotational phase 0.4 noticeable deviations of our measurements from the simple dipole model, which may indicate a more complex topology of the magnetic field structure. On the other hand, as we show later in Sect. 4 the largest dispersion in the hydrogen equivalent width measurements appears around the same phase range and is most likely due to an occultation by circumstellar gas clouds magnetically confined to the magnetic equatorial plane (e.g. Hubrig et al. 2015b). Using the estimate of the stellar radius $R=4.3 \pm 0.3 R_{\odot}$ for a B2 V type star (Harmanec 1988), $v \sin i=270 \pm 20 \mathrm{~km} \mathrm{~s}^{-1}$ (Wisniewski et al. 2015), and the rotation period $P_{\text {rot }}=0.77018 \pm 0.00002 \mathrm{~d}$, we obtain $v_{\text {eq }}=283 \pm 20 \mathrm{~km} \mathrm{~s}^{-1}$ and an inclination angle of the stellar rotation axis to the line of sight $i=73 \pm 19^{\circ}$. From the variation of the phase curve for the field measurements with a mean of $\overline{\left\langle B_{z}\right\rangle}=939 \pm 96 \mathrm{G}$ and an amplitude of $A_{\left\langle B_{\mathrm{Z}}\right\rangle}=1607 \pm 99 \mathrm{G}$, we calculate $\left\langle B_{\mathrm{z}}\right\rangle^{\mathrm{min}}=-669 \pm 139 \mathrm{G}$ and $\left\langle B_{z}\right\rangle^{\max }=2545 \pm 139 \mathrm{G}$. Using the definition by Preston (1967)

$r=\frac{\left\langle B_{\mathrm{z}}\right\rangle^{\min }}{\left\langle B_{\mathrm{z}}\right\rangle^{\max }}=\frac{\cos \beta \cos i-\sin \beta \sin i}{\cos \beta \cos i+\sin \beta \sin i}$,

we find $r=-0.263 \pm 0.05$ and finally following

$\beta=\arctan \left[\left(\frac{1-r}{1+r}\right) \cot i\right]$, 

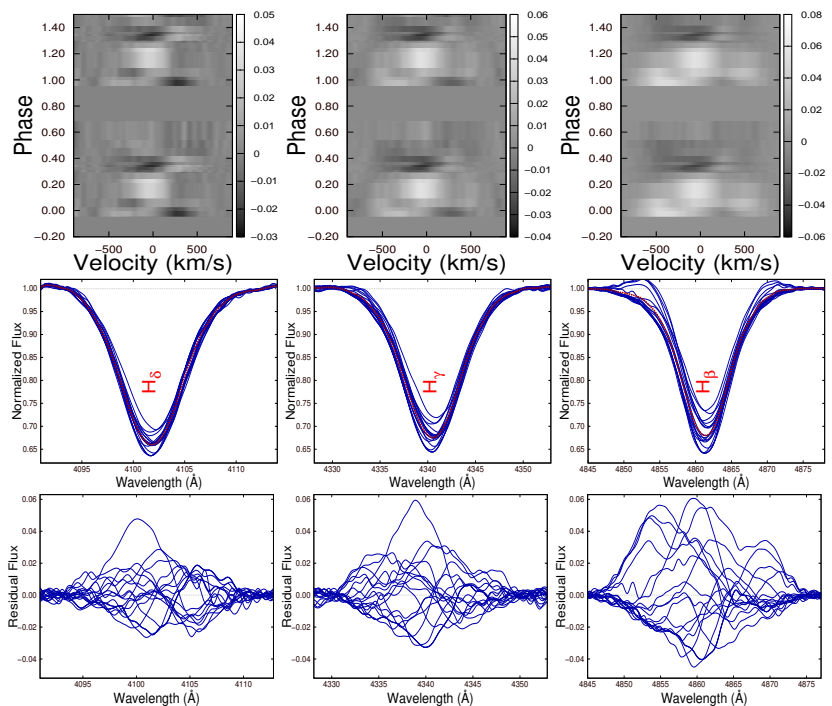

Figure 3. Variability of hydrogen lines in the FORS 2 spectra of HD 345439 over the rotational cycle. The middle and lower panels show the overplotted profiles of the hydrogen lines $\mathrm{H} \delta, \mathrm{H} \gamma$, and $\mathrm{H} \beta$ and the differences between individual and the average line profiles. The upper panel presents the temporal behaviour of these differences. The average profile is shown by the red line.

we calculate the magnetic obliquity angle $\beta=28 \pm 28^{\circ}$.

We can estimate the dipole strength of HD 345439 following the model by Stibbs (1950) as formulated by Preston (1967):

$$
\begin{aligned}
B_{\mathrm{d}} & =\left\langle B_{\mathrm{z}}\right\rangle^{\max }\left(\frac{15+u}{20(3-u)}(\cos \beta \cos i+\sin \beta \sin i)\right)^{-1} \\
& \geqslant\left\langle B_{\mathrm{z}}\right\rangle^{\max }\left(\frac{15+u}{20(3-u)}\right)^{-1} .
\end{aligned}
$$

Assuming a limb-darkening coefficient of 0.3, typical for the spectral type B2V (Claret et al. 2011), we can give a lower limit for the dipole strength of $B_{d} \geqslant 8.98 \pm 0.49 \mathrm{kG}$.

Given the high inclination angle $i=73 \pm 19^{\circ}$ and the low inferred obliquity angle $\beta=28 \pm 28^{\circ}$, both with rather large errors, the estimation of the dipole strength is rather uncertain, leading to $12.7_{-3.7}^{+15.0} \mathrm{kG}$.

\section{SPECTRAL VARIABILITY}

Wisniewski et al. (2015) studied the variability of several He I lines, the $\mathrm{H} \alpha$ line and two Brackett hydrogen lines in the near-infrared (NIR). Although their optical and NIR spectroscopy did not cover the full rotation cycle, the temporary changes in line profiles showed a clear correlation with the rotational phase. As FORS 2 spectra have a much lower spectral resolution, we were able to carry out a variability study using only the strongest lines belonging to three elements: hydrogen, helium, and silicon.

In Fig. 3. we present the overplotted profiles of the hydrogen lines $\mathrm{H} \delta, \mathrm{H} \gamma$, and $\mathrm{H} \beta$, the differences between the individual and average profiles, and the temporal behaviour of these differences in differential dynamic plots. Significant emission in the wings of the hydrogen lines, best visible in the differential dynamic plot of $\mathrm{H} \beta$, appears at the rotational phase around zero, which corresponds to the maximum of the positive magnetic field strength.

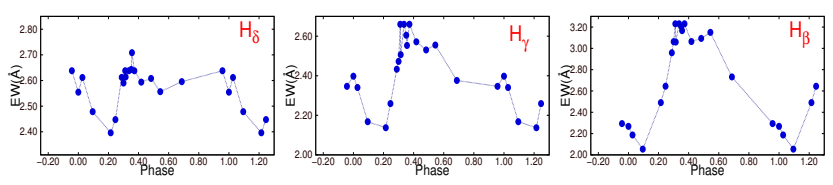

Figure 4. Variability of EWs of hydrogen lines in FORS 2 spectra of HD 345439 obtained at eighteen different rotational phases.

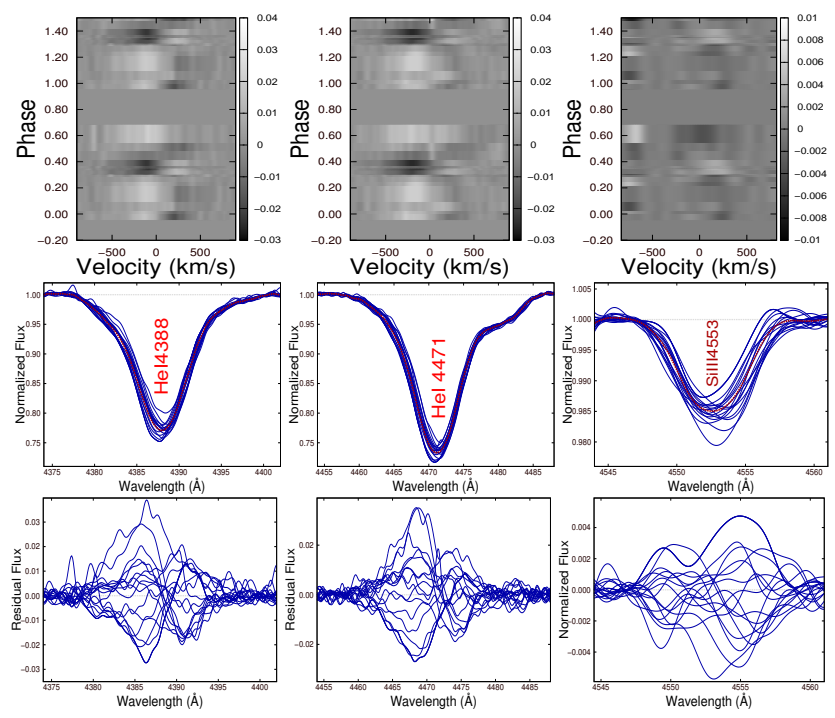

Figure 5. Same as in Fig. 3 but for the helium lines He I 4388, He I 4471, and the silicon line Si III 4553 .

Notably, we observe in the $\mathrm{H} \beta$ emission wings a slight asymmetry, i.e. the blue-shifted emission is somewhat stronger and more extended than the redshifted emission. This behaviour of the $\mathrm{H} \beta$ line differs from the behaviour of the $\mathrm{H} \alpha, \mathrm{Br} 11$, and $\mathrm{Br} \gamma$ lines presented by Wisniewski et al. (2015) in the phase range $0.86-0.18$, indicating a decrease of the blue-shifted emission with increasing wavelength. The phase range $0.86-0.18$ was calculated taking into account the difference in the adopted zero points of ephemeris between the work of Wisniewski et al. (2015) and our work. In Fig.4. we present the variability of the equivalent widths (EWs) of hydrogen absorption lines showing a minimum at rotational phase $0.1-0.2$, which is slightly offset from the positive magnetic pole, and a secondary less pronounced minimum close to the negative magnetic pole. The presence of intensity minima at these phases is likely related to the stronger hydrogen line profile fill-in by the emission presumably originating in the corrotating magnetospheric clouds (e.g. Sundqvist et al. 2012; Hubrig et al. 2015b). As already mentioned in Sect. 3, a large dispersion of EW measurements is detected around the phase 0.4 .

In Fig. 5. we present the overplotted profiles, the differences between the individual and average profiles, and the differential dynamic plots for the helium lines He 4388 and $\mathrm{He}_{\mathrm{I}} 4471$ and the only sufficiently strong silicon line detected in the low-resolution FORS 2 spectra, Si III 4553. Distinct differences are detected in the behaviour between the two elements: He absorption lines are redshifted in the phase ranges from about 0.55 to 0.70 , around the phase 0 , and from 0.1 to 0.2 . In the phase $0.3-0.4$, He lines and the silicon absorption line Si III 4553 are blue-shifted. The offsets to the blue and to the red are indicative of the presence of surface $\mathrm{He}$ and $\mathrm{Si}$ spots similar to the finding of $\mathrm{He}$ and $\mathrm{Si}$ spots on the surface of $\sigma$ Ori E (Reiners et al. 2000). The results of the analysis of 


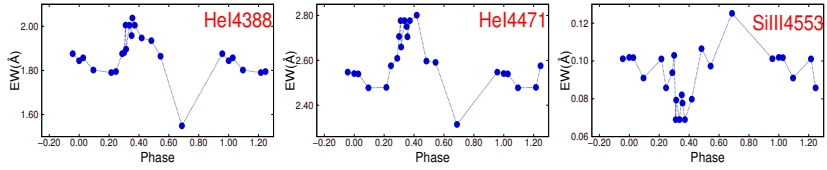

Figure 6. Variability of EWs of He and Si lines in FORS 2 spectra obtained at eighteen different rotational phases.

the variability of EWs of He and Si lines support the presumption of the presence of an inhomogeneous $\mathrm{He}$ and $\mathrm{Si}$ distribution. As is shown in Fig. 6, the Si line strength increases in the phase range from 0.5 to 0.7 , while the intensity of the He lines decreases in the same phase range. For both elements we do not detect any clear correlation with the location of the magnetic poles. The error bars of all presented EW measurements are of the order of the symbol size and less.

\section{DISCUSSION}

Our spectropolarimetric monitoring using FORS 2 at the VLT of the rigidly rotating magnetosphere star HD 345439 revealed the presence of a strong magnetic field with a minimum polar strength of $9 \mathrm{kG}$ reversing over the very short rotation period of $0.77018 \mathrm{~d}$. Both the dipole strength and the very short rotation period of this star are similar to those discovered in two other stars, HR 5907 and HR 7355 with half-day rotation periods (Grunhut et al. 2012; Rivinius et al. 2013), known to belong to the group called the $\sigma$ Ori E analogs (e.g. Groote \& Hunger 1997). Apart from HD 345439, Eikenberry et al. (2014) identified another rigidly rotating magnetosphere star, HD 23478, rotating with a period of $1.04 \mathrm{~d}$ (Jerzykiewicz 1993) and a strong kG magnetic field (Hubrig et al.2015a; Sikora et al.2015). Among the four known very fast rigidly rotating magnetosphere stars, three of them, HD 345439, HD 23478, and HR 5907, show low obliquities of their magnetic axes. For these stars it is expected that the plasma clouds are located close to the equatorial plane (e.g. Townsend \& Owocki 2005). Due to the presence of strong kG magnetic fields and fast rotation, such stars can serve as excellent laboratories to study the magnetic field and element abundance distributions using Zeeman Doppler Imaging, as well as the effect of the magnetic field configuration on the angular momentum loss and the associated spin-down.

The study of the variability of the $\mathrm{He}$ and $\mathrm{Si}$ lines showed the presence of significant chemical abundance variations across the stellar photosphere. However, no clear correlation with the position of the magnetic poles is indicated in our data. Future high-resolution high signal-to-noise spectropolarimetric observations will be worthwhile to determine the locations of these abundance spots as well as the surface distribution of other elements.

Variable emission wings, most clearly detected in the $\mathrm{H} \beta$ line, become stronger at the rotational phase 0 , which corresponds to the best visibility of the positive magnetic pole. The blue-shifted emission appears stronger and more extended than the redshifted emission. This behaviour, which differs from the behaviour of the near-IR lines in HD 345439, was already observed in a few other stars with magnetospheres (e.g., HR 5907 - Grunhut et al. 2012; HR 7355 - Rivinius et al. 2013; HD 23478 - Sikora et al. 2015; CPD $-62^{\circ} 2124$ - Hubrig et al., in preparation). Due to the shortness of the rotation periods and the presence of very strong magnetic fields in the atmospheres of the $\sigma$ Ori $\mathrm{E}$ analogs, these stars are the best candidates to carry out multiwavelength observations at different optical depths to constrain their magnetospheres in more detail (e.g. Carciofi et al. 2013) and to study various atmospheric effects that interact with a strong magnetic field.

\section{ACKNOWLEDGMENTS}

We thank the anonymous referee for useful comments. Based on observations obtained in the framework of the ESO Prg. 097.D0428(A). AK acknowledges financial support from RFBR grant 16-02-00604A. We would like to thank J. R. Lomax for valuable comments on the manuscript.

\section{REFERENCES}

Angel J. R. P., Landstreet J. D., 1970, ApJ, 160, L147

Appenzeller I., et al., 1998, The ESO Messenger, 94, 1

Carciofi A. C., Faes D. M., Townsend R. H. D., Bjorkman J. E., 2013, ApJ, 766, L9

Claret A., Bloemen S., 2011, A\&A, 529, A75

Eikenberry S. S., et al., 2014, ApJL, 784, L30

Groote D., Hunger K., 1997, A\&A, 319, 250

Grunhut J. H., et al., 2012, MNRAS, 419, 1610

Harmanec P., 1988, Bull. Astron. Inst. Czech., 39, 329

Hubrig S., Kurtz D. W., Bagnulo S., Szeifert T., Schöller M., Mathys G., Dziembowski W. A., 2004a, A\&A, 415, 661

Hubrig S., Szeifert T., Schöller M., Mathys G., Kurtz D. W., 2004b, A\&A, 415, 685

Hubrig S., Schöller M., Kholtygin A. F., 2014, MNRAS, 440, L6

Hubrig S., et al., 2015a, A\&A, 578, L3

Hubrig, S., et al., 2015b, MNRAS, 447, 1885

Jerzykiewicz M., 1993, A\&AS, 97, 421

Landstreet J. D., Borra E. F., ApJ, 224, L5

Oksala M. E., Grunhut J. H., Kraus M., Borges Fernandes M., Neiner C., Condori C. A. H., Campagnolo J. C. N., Souza T. B., 2015, A\&A, 578, A112

Press W. H., Teukolsky S. A., Vetterling W. T., Flannery B. P., 1992, Numerical Recipes, 2nd edn. (Cambridge: Cambridge University Press)

Preston G. W., 1967, ApJ, 150, 547

Reiners, A., Stahl, O., Wolf, B., Kaufer, A., Rivinius, T., 2000, A\&A, 363, 585

Rivinius T., Townsend R. H. D., Kochukhov O., Štefl S., Baade D., Barrera L., Szeifert T., 2013, MNRAS, 429, 177

Seber G. A. F., 1977, Linear Regression Analysis (New York: Wiley)

Sikora J., et al., 2015, MNRAS, 451, 1928

Stibbs D. W. N., 1950, MNRAS, 110, 395

Sundqvist, J. O., ud-Doula, A., Owocki, S. P., Townsend, R. H. D., Howarth, I. D., Wade, G. A., 2012, MNRAS, 423, L21

Townsend R. H. D., Owocki S. P., 2005, MNRAS, 357, 251

Wisniewski J. P., et al., 2015, ApJ, 811, L26 